\title{
Vaginal birth after cesarean section - literature review and modern guidelines
}

\author{
Oana-Denisa Bălălău ${ }^{1,2}$, Nicolae Bacalbașa ${ }^{2}$, Octavian Gabriel Olaru ${ }^{1,2}$, Liana Pleș ${ }^{1,2}$, \\ Daniela Anca Stănescu ${ }^{1,2}$
}

${ }^{1}$ BUCUR MATERNITY, ST. IOAN CLINICAL EMERGENCY HOSPITAL, BUCHAREST, ROMANIA

${ }^{2}$ CAROL DAVILA UNIVERSITY OF MEDICINE AND PHARMACY, FACULTY OF MEDICINE, BUCHAREST, ROMANIA

Cesarean section is a major surgical procedure, with significant risks of developing postoperative complications. Consequently, any attempt of vaginal birth after an initial cesarean section (VBCS) aims to avoid such possible complications. The decision on how to give birth in patients with a previous cesarean section, which is complex and based on several factors, should be taken by the pregnant woman together with the obstetrician.

To understand the importance of VBCS, it is first necessary to evaluate its benefits and risks compared to $\mathrm{C}$-section of the uterine scar. By choosing the natural birth route to the detriment of an iterative cesarean section, the potential complications of the latter are avoided. Patients with good labor trial and well monitored have generally better results in terms of complications, both for mother and child. Nevertheless, potential subjects for VBCS should be chosen following rigorous criteria.

As a conclusion, the VBCS decision should be taken by the pregnant woman in consultation with the obstetrician, the medical indications and contraindications being fundamental in choosing the method of birth. The most suitable candidates for the labor test after cesarean section would be those with a low risk of intrapartum uterine rupture. VBCS can decrease the incidence of cesarean section, reducing thus the complications associated with cesarean section for both mother and child.
\end{abstract}

\section{Introduction}

Almost a century ago, obstetricians assumed an idea that guided the choice of birth for women with a history of cesarean section, represented by opinion that 'once cesarean, always cesarean`. Contrary to this perspective, the incidence of vaginal birth after cesarean section (VBCS) increased in time, reaching its peak in 1995-2000 ( $40 \%$ of women with cesarean section), being followed by a steady decline reaching a halving of cases in 2007. Women with a history of cesarean section can choose for a subsequent vaginal birth (VB); in such cases a labor test is required, and can be followed either by vaginal birth or by unplanned or planned cesarean section [1-3].

Cesarean section is a major surgery, with risks of postoperative complications and postoperative recovery time longer than in the case of vaginal birth. The intention to perform the VBCS has the purpose to avoid all these possible complications [4].

The decision for the modality of birth in patients with previous cesarean section is complex and often involves multiple factors that need to be considered. Among these factors, the most important are represented by patient preferences, obstetrical history (history of vaginal births, favorable cervix), data from the literature on the risks and benefits of labor test after caesarean section versus planned cesarean section, and the degree of endowment/ preparation of the hospital for an emergency surgery in case of a negative labor test [5]. American College of Obstetrics and Gynecology guidelines (1999) explicitly recommend that patients undergoing labor after a cesarean section need to be evaluated by obstetrician, anesthesiologist, and medical personnel who may be able to perform an emergency cesarean section in the active phase of labor [6]. 


\section{Discussions}

\section{Benefits of natural childbirth after cesarean section}

By choosing the natural birth route to the detriment of an iterative caesarean section, the potential complications of the surgical procedure are avoided. The benefits of natural birth are multiple, such as avoiding a new uterine incision, a fact that increases the chances of a vaginal birth in the future. In addition, with each scar on the uterine wall the risk of complications of subsequent pregnancies increases, like pregnancy implanted in the uterine scar, placenta praevia, placental fixation abnormalities (placenta accreta/ increta/ percreta) [7, 8]. The blood loss is considerably lower in the case of natural birth, the risk of thrombotic accidents is lower, while the postpartum mobilization is achieved in a much shorter time. The patient's recovery is considerably faster, so that the hospitalization period is also shorter [2,9].

\section{Cesarean delivery - immediate and late postpartum} complications

Cesarean section is a major surgery, accompanied by the risk of postoperative complications. The most common complications are caused by the parietal wound (infection, dehiscence, hematoma, seroma, etc.). Wound infection generally occurs 4-7 days after the surgery, the main risk factors being represented by obesity, chorioamnionitis, blood transfusions, anticoagulant therapy, alcohol or drug use [10].

Postpartum bleeding is an important complication, among the most common causes being uterine atony, placenta accreta, placenta previa, extensive uterine lesions, lesions of the uterine vessels. The risk of postpartum bleeding can be reduced by oxytocic drugs, which are administered after delivery to induce uterine contraction [11].

Cesarean section is the most important risk factor for development of postpartum endometritis, especially when it is performed after the labor onset (emergency intrapartum cesarean section, in the case of a negative labor test). Preoperative antibiotic prophylaxis significantly decreases the incidence of this complication, with one study reporting $11 \%$ for elective cesarean and $28 \%$ for intrapartum emergency cesarean, compared to less than $3 \%$ for vaginal birth $[7,12]$.

The first 6 weeks after childbirth have an increased risk of thromboembolic events for mother, being higher in those who underwent cesarean section (stroke, acute myocardial infarction, venous thromboembolism). A study conducted in 2014 reported a frequency of thrombotic accidents of 246 per 100,000 births by cesarean section in the first 6 weeks postpartum, which is significantly higher than in the case of vaginal births (165 per 100,000 cases) $[8,13]$.
Ovarian vein thrombophlebitis and deep pelvic septic thrombophlebitis are rare complications of cesarean delivery. These two entities have common pathogenic mechanisms and often manifest together, but may differ in clinical manifestations and diagnosis. Patients with ovarian vein thrombophlebitis have fever and abdominal pain located on the affected side, one week after birth. Patients with deep pelvic septic thrombophlebitis usually present few days after cesarean section with fever resistant to antibiotic treatment, in the absence of radiological signs of thrombosis [2, 14]

\section{Cesarean delivery - fetal and neonatal risks}

Even if a cesarean section is performed for the benefit of the fetus, it is still predisposed to risks during the operation. These risks include iatrogenic prematurity and birth trauma, the latter occurring in $1-3 \%$ of cesarean operations and consisting in most cases of mild lacerations related to emergency cesarean delivery $[9,15]$.

In the long run, the cesarean section predisposes to abnormal placentation or abruptio placenta, so that the patient should be monitored by ultrasound carefully in subsequent pregnancies. The risk of placental anomalies increases directly proportional to the number of births by cesarean section $[7,9]$.

Uterine rupture is another possible complication, patients with history of uterine rupture or high vertical hysterotomy (especially at the fundic level) being a category of high risk [5].

Complications that appear at the level of the uterine scar or to the abdominal wall should also be mentioned. Hysterotomy can induce a thinning of the myometrium at the scar site, in the form of a niche, isthmus or uteroperitoneal fistula. The patient may experience postmenstrual spotting, pelvic pain, dysmenorrhea, dyspareunia and even secondary infertility $[2,10]$.

A study conducted in Sweden in 2013 on a cohort of 709,090 women, analyzed the risk of developing endometriosis at the site of the scar after cesarean section. The results revealed a percentage of $0.1 \%$ of patients with this complication. The endometrioma of the incision is generally a soft, palpable mass in the incision that increases in size during menstruation and that is associated with cyclical or continuous pain $[11,16]$.

\section{Maternal risks associated with VBCS}

Uterine rupture - although rare, is the most serious obstetric complication in the case of a labor test after birth by cesarean section. Uterine rupture is defined as total rupture of the uterine wall, including serous. The incidence of this complication is low in developed countries; it is most often associated with negative labor after cesarean section, being life threatening for both mother and fetus [17]. In a 2010 study of a group of 100,000 women who underwent labor, 468 suffered a uterine rupture. It is 
important to mention that the type of incision chosen to the previous cesarean section influences the evolution of a labor sample at a later pregnancy, and also the risk of developing uterine rupture. Inverted T-incision, J-shaped incision or prolonged transverse incision in the upper uterine portion are accompanied by poorer results in terms of VBCS, compared to segmental-transverse hysterotomy, because uterine rupture has a higher prevalence in the first types of incisions (1.9\% compared to $0.7 \%$ ) [12, 13]. There is also an increased risk of uterine rupture in patients with induced labor; data on uterine rupture associated with use of prostaglandins for triggering are suggestive, so that the American College of Obstetrics and Gynecology does not recommend the use of misoprostol for labor induction in women with a history of cesarean section [14].

There are authors who practice imaging measurement of the segment at the level of uterine scar after cesarean section, not only during pregnancy but also during labor in patients who opt for VBCS, to assess the risk of uterine rupture by ultrasound measurement. The thickness of the residual myometrium (at the level of the lower segment) as well as the depth, extent and length of the hypoechoic uterine defects at the level of the uterine scar, all are valuable parameters of predicting the risk of uterine rupture $[15,18]$.

The diagnosis of uterine rupture is established clinically and imaging. This should be suspected in patients undergoing labor after previous cesarean section, who have abnormal fetal heart rhythm (sudden bradycardia or preceded by variable or prolonged decelerations), acute abdominal pain (an increased attention to patients in labor with epidural anesthesia, in which neuraxial analgesia masks pain), vaginal bleeding, hemodynamic instability, cardiotocographic change in the uterine contractility graph and loss of uterine tone, hematuria if the rupture has spread to the bladder $[5,19]$.

Choosing the procedure to surgically resolve the uterine rupture remains a major decision for the obstetrician, who must urgently assess whether the lesion can be repaired conservatively or emergency peripartum hysterectomy is required. The purpose of conservative surgery is to repair the defect of the uterine wall, the control of bleeding, to identify lesions of neighboring organs, to minimize immediate postoperative morbidity and to reduce the risk of complications of subsequent pregnancies. Hysterectomy is indicated when the uterine wall is irreparable or in case of a massive maternal hemorrhage; the decision is taken considering several factors such as the desire for a future pregnancy, the extension of uterine lesions, the patient's hemodynamic status and the operator's experience in resolving complex uterine lesions [5, 20-22].

A literature review of a sample consisting on 142,075 patients, who underwent labor after a cesarean section and which ended in 880 women through uterine rupture, reported need of hysterectomy and erythrocyte mass transfusion as the most common therapeutic measures [16].

The maternal infectious risks in case of VBCS are significantly lower comparing to cesarean section, being represented especially by chorioamniotitis and postpartum endometritis. Chorioamniotitis is usually associated with prolonged labor and ruptured membranes for a long period of time. Patients with multiple digital vaginal examinations, those with cervical incompetence, meconium amniotic fluid, nulliparity, genital infections such as sexually transmitted diseases, group B Streptococcus infection and bacterial vaginitis also present an increased risk for chorioamniotitis [17].

Vaginal birth is associated with long-term risk for pelvic floor lesions, such as genital prolapse, urinary incontinence, and fecal incontinence. Pregnancy and childbirth would contribute to appearance of these lesions by compression, stretching or rupture of nerves, muscles or connective tissue. In turn, an intact neuromuscular function and the support of the pelvic organs seem to be critical for the normal location/ functioning of the pelvic organs [18].

\section{Fetal risks associated with VBCS}

Transient tachypnea of the newborn is an important complication, which is characterized by pulmonary edema resulting from delayed resorption and clearance of fetal alveolar fluid. It is a relatively benign condition, but there are data that correlate it with an increased risk for the newborn to develop wheezing afterwards, during childhood [19].

Perinatal and neonatal mortality rates were higher in patients with VBCS following labor, than in those who gave birth by elective cesarean section $[1,23]$.

A study conducted on 15,338 full-term pregnant women who underwent labor testing reported 2 neonatal deaths and 7 cases of hypoxic-ischemic encephalopathy, with rates of 1.4 per 10,000 and 4.6 per 10,000 , respectively. There were 114 cases of uterine rupture, of which $1.8 \%$ resulted in neonatal death while $6.2 \%$ conducted to hypoxic-ischemic encephalopathy [20].

\section{The indications of $\mathrm{VBCS}$}

The ideal case of a positive labor test is the patient who have a low transverse uterine incision at the previous cesarean section. There is consistent evidence to support this observation, as these patients have the lowest risk of scarring during labor. Studies report percentages of about $0.4-0.6 \%$ uterine ruptures in these cases [21].

Other potential candidates are those with a low vertical uterine incision. If the vertical incision is prolonged in the contractile portion of the uterus, it is considered a classic hysterotomy and thus a possible VBCS is excluded. There are studies sustaining that a labor test is possible even after two cesarean operations with low transverse uterine incisions, the success rates being comparable to cases with a single low transverse hysterotomy (65\% vs. $75 \%$ ) [22]. 
If the pregnant woman has had a previous vaginal birth, before or after the cesarean section, the chances of having VBCS without complications are significantly increased [24].

There are a number of cases that are not contraindicated by VBCS, according to the American College of Obstetrics and Gynecology (ACOG), several studies supporting these practices. During pregnancy, after 40 weeks of gestation the labor test can be tried, and the pregnant woman is carefully monitored so that the risk of failure of the sample is calculated (including the occurrence of obstetric complications). There are no significantly higher risks in such pregnancies, comparable to cases under 40 weeks. However, there are delays related to the onset of labor after 40 weeks, with some studies reporting higher rates of uterine rupture and other possible complications compared to pregnancies under 40 weeks [23]. The twin pregnancy can also be a candidate for VBCS, if the pregnant woman has a low transverse uterine incision at the previous cesarean section, the frequency of a positive labor test being close to those of a single fetal pregnancy [24]. Pregnancies with macrosomal fetuses (> 4000g) can be considered as a VBCS option. In these cases, the obstetrician have to carefully analyze the patient's obstetrical history (weights of girls from previous births, vaginal birth history) and, if the pregnant woman has had a previous natural birth, then the probability of a positive labor test increases significantly. If the indication for cesarean section at the previous pregnancy were represented by cephalopelvin disproportion with failure of labor progression, then the success rates of the labor sample are low [1]. A published study including patients with macrosomal fetuses and divided in distinct groups by weight: <4000g, 4000-4249g, 4250g-4500g, and $>4500 \mathrm{~g}$, reported for VBCS the following probability rates: $68 \%$, $52 \%, 45 \%$, and $38 \%$ respectively [25].

\section{The contraindications of $V B C S$}

The standard contraindications of labor and vaginal birth apply in a similar way to the case of VBCS (complete placenta praevia, placenta accreta/ increta/ percreta, dystocic presentation, cephalopelvic disproportion) [16].

Patients with a history of fundic incision (cesarean section, myomectomy, uterine reconstruction following a congenital malformation) are not recommended for VBCS. There are studies showing that classical or T-shaped uterine incisions are more frequently followed by uterine rupture during labor than the low transverse hysterotomies [26].

A history of uterine rupture raises significant risks of recurrence for pregnant women during labor (with an incidence of $6 \%$ ). The risk factors mentioned in the literature are represented by oxytocin abuse and dystocic presentations. As these patients have an increased obstetric risk, it is preferable not to recommend additional pregnancies to these people $[27,28]$. If the patient wants however a pregnancy, the caesarean section is recommended at 36-37 weeks, to avoid the onset of labor that can lead to recurrence of rupture. The obstetrical history is important for decision related to the moment of birth, which must be chosen according to the particularity of each case (premature labor in the current pregnancy, the history of premature rupture of the uterus or antepartum in a previous pregnancy) [5].

\section{Conclusions}

The VBCS decision should be taken by the pregnant woman together with the obstetrician, through a close monitoring of the labor test and a full understanding of the indications and contraindications of VBCS. Cesarean section is a major surgery, with significant risks of postoperative complications; the option of VBCS is intended to avoid all these possible complications. In selected cases VBCS decreases the need for a cesarean section, proving thus that is able to reduce complications for mother and child following this type of birth.

\section{Conflict of interest disclosure}

There are no known conflicts of interest in the publication of this article. The manuscript was read and approved by all authors.

\section{References}

1. Miazga E, Reed C, Tunde-Byass M, Cipolla A, Shapiro J, Shore EM. Decreasing Cesarean Delivery Rates Using a Trial of Labour After Cesarean (TOLAC) Bundle. J Obstet Gynaecol Can. 2020; S17012163(20)30217-6. doi:10.1016/j.jogc.2020.02.113

2. Kwong FL, Hamoodi I. Postnatal diagnosis of an occult uterine scar dehiscence after three uncomplicated vaginal births after Caesarean section: A case report. Case Rep Womens Health. 2020; 27: e00203. doi: 10.1016/j.crwh.2020.e00203

3. Metz TD, Scott JR. Contemporary management of VBAC. Clin Obstet Gynecol. 2012;55(4):1026-1032. doi:10.1097/GRF.0b013e318261a2ad

4. Grinstead J, Grobman WA. Induction of labor after one prior cesarean: predictors of vaginal delivery. Obstet Gynecol. 2004; 103(3): 534-538. doi:10.1097/01.AOG. 0000115508.75370 .23

5. Landon MB, Lynch CD. Optimal timing and mode of delivery after cesarean with previous classical incision or myomectomy: a review of the data. Semin Perinatol. 2011;35(5):257-261.

doi:10.1053/j.semperi.2011.05.008

6. Sargent JA, Savitsky LM, Dissanayake MV, Lo JO, Cheng YW, Caughey AB. Gestational Weight Gain during Pregnancy as an Important Factor Influencing a 
Successful Trial of Labor following Two Previous Cesareans. Am J Perinatol. 2019;36(6):588-593. doi: 10.1055/s-0038-1670679

7. Smaill FM, Grivell RM. Antibiotic prophylaxis versus no prophylaxis for preventing infection after cesarean section. Cochrane Database Syst Rev. 2014; (10):CD007482. Published 2014 Oct 28. doi: 10.1002/14651858.CD007482.pub3

8. Burrows LJ, Meyn LA, Weber AM. Maternal morbidity associated with vaginal versus cesarean delivery. Obstet Gynecol. 2004;103(5 Pt 1):907-912. doi:10.1097/01.AOG.0000124568.71597.ce

9. Kamel H, Navi BB, Sriram N, Hovsepian DA, Devereux RB, Elkind MS. Risk of a thrombotic event after the 6-week postpartum period. $N$ Engl J Med. 2014;370(14):1307-1315. doi:10.1056/NEJMoa1311485

10. Alexander JM, Leveno KJ, Hauth J, et al. Fetal injury associated with cesarean delivery. Obstet Gynecol. 2006;108(4):885-890. doi:10.1097/01.AOG.0000237116.72011.f3

11. Antila-Långsjö RM, Mäenpää JU, Huhtala HS, Tomás EI, Staff SM. Cesarean scar defect: a prospective study on risk factors. Am J Obstet Gynecol. 2018; 219(5): 458.e1-458.e8. doi:10.1016/j.ajog.2018.09.004

12. Andolf E, Thorsell M, Källén K. Caesarean section and risk for endometriosis: a prospective cohort study of Swedish registries. BJOG. 2013;120(9):1061-1065. doi:10.1111/1471-0528.12236

13. Guise JM, Denman MA, Emeis C, et al. Vaginal birth after cesarean: new insights on maternal and neonatal outcomes. Obstet Gynecol. 2010;115(6):1267-1278. doi:10.1097/AOG.0b013e3181df925f

14. Landon MB, Hauth JC, Leveno KJ, et al. Maternal and perinatal outcomes associated with a trial of labor after prior cesarean delivery. $N$ Engl J Med. 2004; 351(25): 2581-2589. doi:10.1056/NEJMoa040405

15. ACOG Practice Bulletin No. 205: Vaginal Birth After Cesarean Delivery. Obstet Gynecol. 2019;133(2):e110e127. doi:10.1097/AOG.0000000000003078

16. Naji O, Abdallah Y, Bij De Vaate AJ, et al. Standardized approach for imaging and measuring Cesarean section scars using ultrasonography. Ultrasound Obstet Gynecol. 2012;39(3):252-259. doi:10.1002/uog.10077

17. Chauhan SP, Martin JN Jr, Henrichs CE, Morrison JC, Magann EF. Maternal and perinatal complications with uterine rupture in 142,075 patients who attempted vaginal birth after cesarean delivery: A review of the literature. Am J Obstet Gynecol. 2003;189(2):408-417. doi:10.1067/s0002-9378(03)00675-6

18. Levin G, Meyer R, Mor N, et al. Trial of Labor After Cesarean in Adolescents - A Multicenter Study. $J$
Pediatr Adolesc Gynecol. 2020; S10833188(20)30159-5. doi:10.1016/j.jpag.2020.02.006

19. Li WY, Liabsuetrakul T, Stray-Pedersen B. Effect of mode of delivery on perceived risks of maternal health outcomes among expectant parents: a cohort study in Beijing, China. BMC Pregnancy Childbirth. 2014;14:12. doi:10.1186/1471-2393-14-12

20. Familiari A, Neri C, Caruso A, et al. Vaginal birth after caesarean section: a multicentre study on prognostic factors and feasibility. Arch Gynecol Obstet. 2020;301(2):509-515. doi:10.1007/s00404-020-054540

21. Dooley J, Jumah N, Okenden H, et al. Caesarean Sections and Vaginal Birth After Caesarean Section Rates in a First Nations Community-Based Obstetrical Program in Northwest Ontario. J Obstet Gynaecol Can. 2020;S1701-2163(19)30768-6. doi:10.1016/j.jogc.2019.08.024

22. Asgarian A, Rahmati N, Nasiri F, Mohammadbeigi A. The Failure Rate, Related Factors, and Neonate Complications of Vaginal Delivery after Cesarean Section. Iran J Nurs Midwifery Res. 2019;25(1):65-70. doi:10.4103/ijnmr.IJNMR_101_19

23. Metz TD, Allshouse AA, Faucett AM, Grobman WA. Validation of a vaginal birth after cesarean delivery prediction model in women with two prior cesarean deliveries. Obstet Gynecol. 2015;125(4):948-952. doi:10.1097/AOG.0000000000000744

24. Hammoud A, Hendler I, Gauthier RJ, Berman S, Sansregret A, Bujold E. The effect of gestational age on trial of labor after Cesarean section. J Matern Fetal Neonatal Med. 2004; 15(3): 202-206. doi: 10.1080/ 14767050410001668329

25. Varner MW, Thom E, Spong CY, et al. Trial of labor after one previous cesarean delivery for multifetal gestation. Obstet Gynecol. 2007;110(4):814-819. doi: 10.1097/01.AOG.0000280586.05350.9e

26. Elkousy MA, Sammel M, Stevens E, Peipert JF, Macones $\mathrm{G}$. The effect of birth weight on vaginal birth after cesarean delivery success rates. Am J Obstet Gynecol. 2003;188(3):824-830. doi: $10.1067 / \mathrm{mob} .2003 .186$

27. Sabol B, Denman MA, Guise JM. Vaginal birth after cesarean: an effective method to reduce cesarean. Clin Obstet Gynecol. 2015;58(2):309-319.

doi:10.1097/GRF.0000000000000101

28. Chibber R, El-Saleh E, Al Fadhli R, Al Jassar W, Al Harmi J. Uterine rupture and subsequent pregnancy outcome--how safe is it? A 25-year study. J Matern Fetal Neonatal Med. 2010; 23(5): 421-424. doi: $10.3109 / 14767050903440489$ 